\title{
Incoherent magnetization rotation observed in subnanosecond time-resolving x-ray photoemission electron microscopy
}

\author{
C. M. Schneider ${ }^{\text {a) }}$ and A. Kuksov \\ Institut f. Festkörperforschung IFF-6, Forschungszentrum Jülich, D-52425 Jülich, Germany, \\ and Institut f. Festkörper- u. Werkstoffforschung Dresden, D-01069 Dresden, Germany \\ A. Krasyuk, A. Oelsner, D. Neeb, S. A. Nepijko, and G. Schönhense \\ Institut f. Physik, J.-Gutenberg Universität, D-55099 Mainz, Germany \\ I. Mönch and R. Kaltofen \\ Institut f. Festkörper- und Werkstoffforschung Dresden, D-01069 Dresden, Germany \\ J. Morais \\ Instituto de Fisica-UFRGS, Porto Alegre, RS, 91501-970, Brazil \\ C. de Nadaï and N. B. Brookes \\ European Synchrotron Radiation Facility, BP 220, F-38043 Grenoble, France
}

(Received 15 March 2004; accepted 8 July 2004)

\begin{abstract}
We present recent results of time-resolved x-ray photoemission electron microscopy on permalloy microstructures. The stroboscopic experiments feature a time-resolution of $\Delta \tau \leqslant 130 \mathrm{ps}$. We observe a strong influence of incoherent magnetization rotation processes, leading to a significant transient stray-field formation at the edges of the microstructure. (C) 2004 American Institute of Physics.
\end{abstract}

[DOI: $10.1063 / 1.1790606$ ]

A solid understanding of the micromagnetic processes which determine the dynamics of magnetization reversal is mandatory for a further improvement of existing devices and the evaluation of advanced magnetic writing strategies. These processes comprise domain nucleation, domain-wall motion, or incoherent and coherent magnetization rotation. Although some knowledge can be gained from spatially integrating over the sample response, a much more detailed picture will emerge from laterally resolving techniques. Direct imaging of fast magnetization reversal remains a considerable experimental challenge and has long been a domain of magnetooptical techniques. ${ }^{1-4}$ However, the recent years have witnessed the advancement of powerful magnetic microscopy techniques based on the use of synchrotron radiation. ${ }^{5,6}$ In addition to a high magnetic contrast arising from magnetic x-ray circular dichroism (MXCD), they combine high lateral resolution with chemical selectivity and provide either high surface sensitivity (x-ray photoemission electron microscopy, XPEEM) or high information depth ( $\mathrm{x}$ ray transmission microscopy). The successful implementation of nanosecond time resolution into XPEEM was demonstrated just recently. ${ }^{7-9}$

In this contribution we report on stroboscopic XPEEM studies on permalloy microstructures with a time resolution of $130 \mathrm{ps}$. The images exhibit a yet unobserved richness of detail, which is attributed to incoherent magnetization rotation processes. These processes lead to strong transient strayfield contributions at the edges of the microstructures.

The time-resolved (TR)-XPEEM experiments have been performed with circularly polarized soft x-rays from the elliptical undulator beamline ID-08 at the European Synchrotron Radiation Facility (Grenoble, France) using a dedicated photoemission electron microscope of the IS-PEEM type. ${ }^{10}$

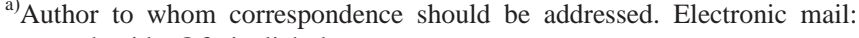
c.m.schneider@fz-juelich.de
The instrument features a dual image detection system: a standard imaging unit (phosphor screen and 12-bit slow scan CCD camera) and a two-dimensional delay-line detector ${ }^{11}$ for low-signal applications. This delay-line detector was particularly employed in previous experiments using low-flux single-bunch operational mode. The experiments described below employed ESRF's "16-bunch" mode, which delivers light pulses of about $106 \mathrm{ps}$ width and about $176 \mathrm{~ns}$ repetition time. A bunch marker generated from the rf accelerating the electrons in the storage ring was used to trigger the magnetic-field pulse. The time delay between field and light pulse was set by a switchable delay unit. For a sufficient signal-to-noise ratio, each image was averaged over about $10^{7}$ subsequent remagnetization events.

The samples comprised a $\mathrm{Cu}$ microstripline of $100 \mu \mathrm{m}$ width and $200 \mathrm{~nm}$ thickness. The magnetic sample was placed on top of the stripline and consisted of a bilayer (40 $\mathrm{nm} \mathrm{Ni}{ }_{81} \mathrm{Fe}_{19}, 2 \mathrm{~nm} \mathrm{Cu}$ ), which had been lithographically patterned into microstructures of different geometrical shapes and sizes (see also Ref. 7). Passing a short current pulse through the microstripline generated a magnetic field at the position of the microstructures. In this letter we focus on rectangular elements (bars) of $20 \times 80 \mu \mathrm{m}^{2}$ and 40 $\times 80 \mu \mathrm{m}^{2}$ size.

The attainable time resolution $\Delta \tau$ is not only given by the light pulse width $(\Delta T=106 \mathrm{ps})$, but also the jitter between bunchmarker and magnetic field pulse. From experimentally determined values, we estimate $\Delta \tau \leqslant 130 \mathrm{~ns}$. In Fig. 1 we show a sequence of domain images (Ni $L_{3}$ MXCD contrast) taken along the rising edge of a unipolar field pulse $(\Delta B / \Delta t \sim 1 \mathrm{mT} / \mathrm{ns})$ with a delay step width of $\Delta t=125 \mathrm{ps}$. Prior to the field pulse, both elements reveal Landau-like (flux-closure dominated) domain patterns. ${ }^{11,12}$ This important finding proves the sample to reproducibly relax back into the same state after each field pulse cycle. Obviously, the demagnetizing field takes the role of the static bias field applied 


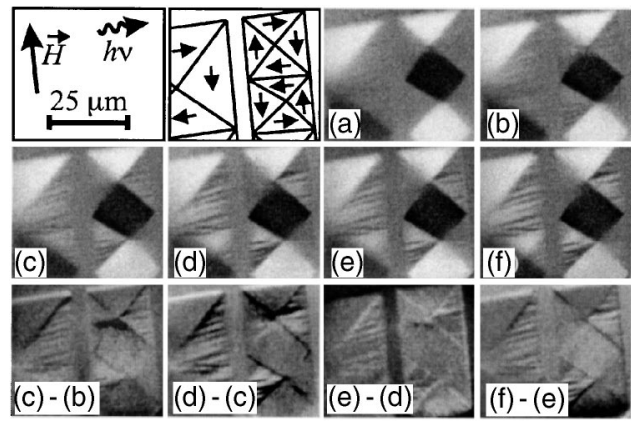

FIG. 1. TR-XPEEM domain images of $\mathrm{Ni}_{81} \mathrm{Fe}_{19}$ rectangular bars taken along the rising edge of a unipolar magnetic field pulse at $t=0$ (a), 125 (b), 250 (c), 375 (d), 500 (e), and 625 ps (f). Directions of light incidence (projection on sample surface) and remagnetization field $\vec{H}$ are given, together with a sketch of the particle domain structure in the field free state. Bottom row: subsequent differences between images (b) and (f).

in previous experiments. ${ }^{1}$ With increasing strength of the transient field, we start to see characteristic changes in the domain configuration. These changes are highlighted by forming differences between subsequent images (bottom row of Fig. 1). The difference images exhibit distinct structures indicating an actual time resolution well below the chosen delay step width.

We note that the dominant changes in Fig. 1, namely the formation of stripelike domains, appear alternatingly in the triangular domains on the right- and left-hand side of the bar, respectively. In the static case, these domains have a magnetization vector $\vec{M}$ opposite to the direction of the transient field $\vec{H}(t)$ generated by the current pulse through the stripline. Domains with $\vec{M} \| \vec{H}(t)$ exhibit only a homogeneous contrast level. A magnetic-field pulse of opposite sign creates the stripelike pattern in the opposing triangles (not shown). This clearly demonstrates that the orientation between $\vec{M}$ and $\vec{H}(t)$ is responsible for this behavior. A similar observation is made with a bipolar pulse (Fig. 2). After the transient field $\vec{H}(t)$ changed sign, the stripelike triangular areas have moved from the right- to the left-hand side of the rectangles and vice versa [compare Figs. 2(b) and 2(d)]. A closer inspection of Fig. 2(c) reveals, however, that the bipolar pulse excitation is more complicated, as stripes are formed in the opposing triangular domains already prior to $\vec{H}(t)$ changing sign. This is due to a slow decay of the stripe phase (see below). We also note that at the bottom of the wider rectangle the entire do-

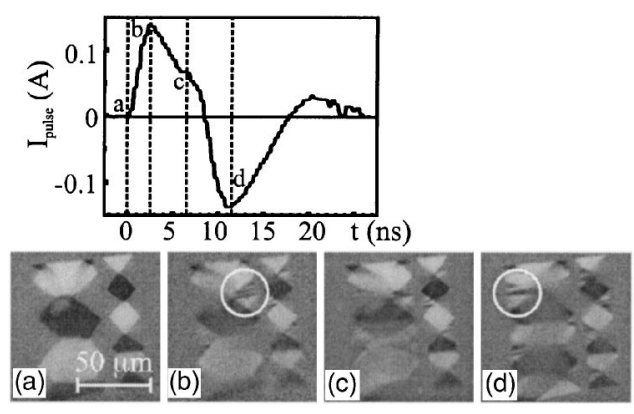

FIG. 2. Domain images mapping a bipolar magnetic-field pulse (top panel) at $t=0$ (a), 2.5 (b), 6.5 (c), and $11.5 \mathrm{~ns}$ (d). Areas with stripelike domains in the upper part of the wider bar are marked by circles.

Downloaded 21 Dec 2006 to 134.94.122.39. Redistribution subject to AIP license or copyright, see http://apl.aip.org/apl/copyright.jsp

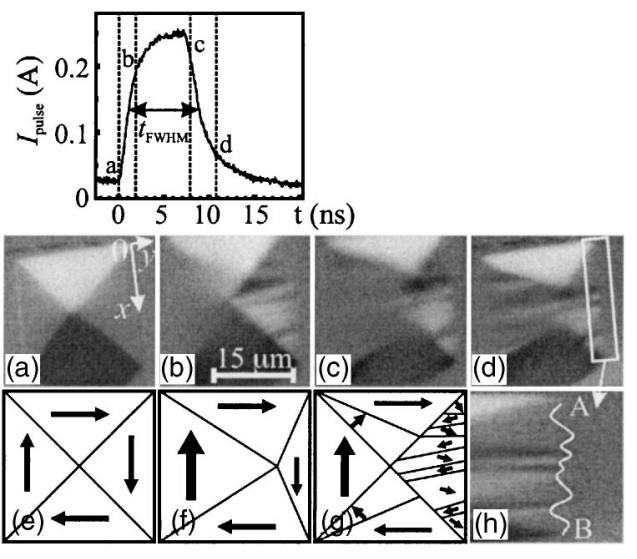

FIG. 3. Magnetic domain images of the upper part of the narrow rectangle in Fig. 1 mapping a unipolar field pulse (top panel) at $t=0$ (a), 1.750 (b), 7.875 (c), and $10.875 \mathrm{~ns}(\mathrm{~d})$. Area marked by a rectangle in (d) is stretched $\times 5$ in horizontal direction (h). Schematic images of expected behavior of the Landau structure (e) in a slowly varying field (f) and formation of a stripelike structure at $t=7.875 \mathrm{~ns}$ after the leading edge of the pulse (g). The white line in (h) traces the deformation profile along the right edge $A B$ of the particle.

main pattern changes, if $\vec{H}(t)$ has taken a negative polarity [Fig. 2(d)].

Images taken at smaller field of view reveal more details of the dynamic behavior. Figure 3 shows the domains in the upper part of the narrow bar at $t=0$ (a), 1.75 (b), 7.875 (c), and $10.875 \mathrm{~ns}$ (d) after the onset of a positive magnetic-field pulse (upper panel). The Landau pattern $[\vec{M}$ indicated by the arrows in Fig. 3(e)] observed at $t=0$ [Fig. 3(a)] proves the magnetization to fully relax within the repetition time (176 ns). Figure 3(f) shows the change to be expected during a slow remagnetization event. The applied field $\vec{H}$ will move the domain walls in the Landau pattern, expanding the domain with $\vec{M} \| \vec{H}$ on the expense of the others. In particular, the domain with $\vec{M}$ antiparallel to $\vec{H}(t)$ will shrink and the vortex in the center of Fig. 3(e) will shift to the right. Also in the pulsed-field case, the domain with $\vec{M} \| \vec{H}(t)$ grows in the applied field, while the domains with $\vec{M} \perp \vec{H}(t)$ shrink. The domain with $\vec{M}$ antiparallel to $\vec{H}(t)$, however, breaks up into stripes, which cover almost the same area as the original domain. As a consequence, the vortex splits up into at least two vortices, which move along the initial position of the domain walls with inceasing field strength. Reconstructing the magnetization distribution from the gray levels in Fig. 3(c) reveals the complicated transient domain pattern [Fig. $3(\mathrm{~g})]$.

We also remark that the decay of this excited state takes a different path [Fig. 3(d)]. Only upon reduction of $\vec{H}$ does the stripe-domain pattern already expand into the left-hand side of the bar, whereas for the top and bottom domains with $\vec{M} \perp \vec{H}$, shape and size change only marginally even at $H(t)<0.2 \cdot H_{\max }$. This slow decay consistently explains the observation in the case of the bipolar pulse. Unfortunately, a discussion of the decay mechanisms exceeds the scope of this paper.

During the fast reversal process, shape and arrangement of the magnetic domains are nonstationary and stray fields may arise outside the particle. They show up in the XPEEM image as deformation of the particle edges. ${ }^{13}$ Figure $3(\mathrm{~h})$ 


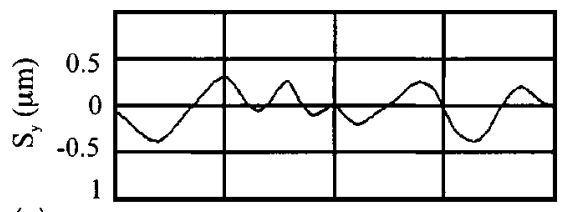

(a)

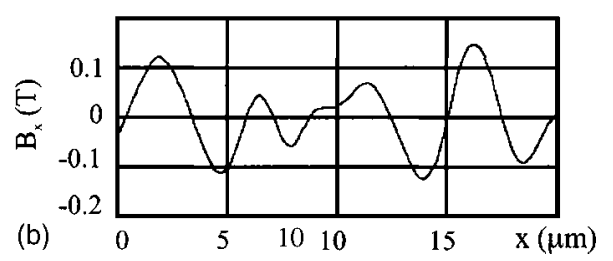

FIG. 4. Stray-field induced shift of the particle's right edge $S_{y}$ and calculated variation of magnetic induction along this edge on the object surface $B_{x}$.

displays an enlarged view of the edge region [rectangle in Fig. 3(d)]. To visualize the small deformations $S_{y}(x)$ of the edge giving rise to a wavelike path $A B$, the image in Fig. 3(h) has been horizontally stretched five times. From the shift of the particle edge $S_{y}(x)$ observed along the $y$ axis [Fig. 4(a)], we can determine the magnetic induction $B_{x}(x)$ along the edge $A B$ at the object surface [Fig. 4(b)]. ${ }^{13}$ We find transient stray-field components perpendicular to the particle edge as high as $0.1 \mathrm{~T}$.

A possible explanation for our findings can be drawn from the work of the Freeman group ${ }^{14,15}$ using time-resolved Kerr microscopy and micromagnetic simulations. The authors investigated permalloy microstructures of similar size. These were saturated in a static field, with the pulsed magnetic field applied in the opposite direction. Above certain field strengths, the authors observed the appearance of complex stripelike domain phases. This behavior was attributed to incoherent rotation processes. We believe that a similar mechanism holds in our case, if we assume that the demagnetizing field which stabilizes the Landau structure acts similar to the guiding field in the time-resolved Kerr microscopy experiments of Freeman et al. and Hiebert et al. ${ }^{14,15}$ In an incoherent rotation process, the homogeneous magnetization breaks up into a system of elongated regions, in each of which the local magnetization vector rotates into an orientation close to $\vec{M} \perp \vec{H}(t)$. The rotation is not restricted to the film plane, as the transient domain patterns observed by the Freeman group revealed also sizable components of $\vec{M}$ nor- mal to the film plane. Probing these components will be an interesting aspect in future vectorial TR-XPEEM investigations. The microscopic origin of this incoherent rotation is not yet fully understood. We suggest, however, that it may be associated with structural (edge roughness) or magnetic inhomogeneities (magnetization ripple) of the sample. The first is introduced by the lithographic patterning process and may create nucleation centers for the stripe formation at the sample edge. The second depends on the film thickness. Further high-resolution studies as a function of film thickness and feature size are needed to clarify this situation.

The authors are indebted to C. Krien, I. Fiering, B. Eichler, S. Sieber, and V. Matthes for expert technical support. Financial support by the BMBF through Grant Nos. 05 KS1 BDA/9 and $05 \mathrm{KS} 1 \mathrm{UM} 1 / 5$ is gratefully acknowledged.

${ }^{1}$ M. R. Freeman and W. K. Hiebert, in Spin Dynamics in Confined Magnetic Structures I, edited by B. Hillebrands and K. Ounadjela (Springer, Berlin, 2002).

${ }^{2}$ A. Kirilyuk, J. Ferré, J. Pommier, and D. Renard, J. Magn. Magn. Mater. 121, 536 (1993).

${ }^{3}$ R. P. Cowburn, J. Ferré, S. J. Gray, and J. A. C. Bland, Phys. Rev. B 58, 11507 (1998).

${ }^{4}$ B. C. Choi, G. E. Ballentine, M. Belov, W. K. Hiebert, and M. R. Freeman, Phys. Rev. Lett. 86, 728 (2001).

${ }^{5}$ C. M. Schneider and G. Schönhense, Rep. Prog. Phys. 65, R1785 (2002).

${ }^{6}$ P. Fischer, T. Eimüller, G. Schütz, G. Schmahl, P. Guttmann, and D. Raasch, in X-Ray Microscopy and Spectromicroscopy, edited by J. Thieme, G. Schmahl, D. Rudolph, and E. Umbach (Springer, Berlin, 1998).

${ }^{7}$ A. Krasyuk, A. Oelsner, S. A. Nepijko, A. Kuskov, C. M. Schneider, and G. Schönhense, Appl. Phys. A: Mater. Sci. Process. 76, 863 (2003).

${ }^{8}$ J. Vogel, W. Kuch, M. Bonfim, J. Camarero, Y. Pennec, F. Offi, K. Fukumoto, J. Kirschner, A. Fontaine, and S. Pizzini, Appl. Phys. Lett. 82, 2299 (2003).

${ }^{9}$ S.-B. Choe, Y. Acremann, A. Scholl, A. Bauer, A. Doran, J. Stöhr, and H. A. Padmore, Science 304, 420 (2004).

${ }^{10} \mathrm{See}, \mathrm{http}: / /$ www.focus-gmbh.com

${ }^{11}$ A. Oelsner, O. Schmidt, M. Schicketanz, M. Klais, G. Schönhense, V. Mergel, O. Jagutzki, and H. Schmidt-Böcking, Rev. Sci. Instrum. 72, 3968 (2001).

${ }^{12}$ A. Hubert and R. Schäfer, Magnetic Domains: The Analysis of Magnetic Microstructures (Springer, Berlin, 1998).

${ }^{13}$ S. A. Nepijko, M. Klais, A. Oelsner, O. Schmidt, G. H. Fecher, G. Schönhense, U. Muschiol, C. M. Schneider, S. Zennaro, N. Zema, and N. N. Sedov, Appl. Phys. A: Mater. Sci. Process. 83, 295 (2002).

${ }^{14}$ M. R. Freeman, W. K. Hiebert, and A. Stankiewicz, J. Appl. Phys. 82, 6217 (1998).

${ }^{15}$ W. K. Hiebert, G. E. Ballentine, L. Lagae, R. W. Hunt, and M. R. Freeman, J. Appl. Phys. 92, 392 (2002). 